\title{
EVALUATION OF ZUCKERMAN-KUHLMAN PERSONALITY QUESTIONNAIRE-50-CC IN SERBIAN CULTURE'
}

\section{Dušanka Mitrović², Petar Čolović and Snežana Smederevac}

Department of Psychology, University of Novi Sad, Serbia

\begin{abstract}
The main purpose of this research was to evaluate dimensions of the Zuckerman-Kuhlman Personality Questionnaire-50-CC (ZKPQ-50-CC) in the Serbian culture in the light of some of the basic assumptions of the alternative five-factor model - the cross-cultural stability, construct validity and heritability. The study had two parts. The aim of the first study is to evaluate the replicability of the alternative FFM in a Serbian sample $(N=$ 1155). Three structural models were tested using confirmatory factor analysis. The model which included correlated dimensions and four omitted items showed the best fit. The second study, conducted on a sample of 200 participants (90 male and 110 female), was set to test several models of relations between the alternative FFM and Eysenck's PEN model. Four models were tested using the structural equation modeling. The best- fitting model included three latent dimensions, which corresponded to PEN dimensions.
\end{abstract}

Keywords: alternative FFM, ZKPQ-5-CC, cross-cultural replicability, PEN model

\footnotetext{
${ }^{1}$ The research was carried out within the Project "Psychological Characteristics of the Society in Transition" supported by the Ministry of Science of the Republic of Serbia.

2E-mail: dudamit@eunet.rs
} 


\section{Introduction}

Beginning in the late 1980s, Zuckerman and Kuhlman (Zuckerman et al., 1993) focused on a development of a questionnaire for the assessment of the basic personality dimensions. After a series of studies, they constructed the Zuckerman-Kuhlman Personality Questionnaire (ZKPQ; Zuckerman, 2002), which included five dimensions: Activity (Act), Aggressiveness - Hostility (Agg-Host), Impulsive Sensation Seeking (ImpSS), Neuroticism - Anxiety (NAnx) and Sociability (Sy). The development of the alternative FFM was guided by specific criteria, with the aim to identify the traits that can be considered as basic ones (Zuckerman, 1991). The first of those criteria is the request that the basic dimensions of personality must be reliably identified across different methods, genders, ages, and cultures. The ZKPQ (Zuckerman, 2002) has been validated in different cultures, such as German (Angleitner et al., 2004), Chinese (Wu et al., 2000), and Spanish (Aluja et al., 2002).

The reliability of the ZKPQ scales has ranged from 0.68 to 0.81 . Gradually, a shortened form, which consists of 50 items selected according to the standard psychometric criteria, was developed (ZKPQ-50-CC; Aluja et al., 2006) for the purposes of the cross-cultural validation. The validation in the American, German, Spanish and Swiss cultures showed good structural and psychometric properties of the ZKPQ-50-CC (Aluja et al., 2006), and correlations of the shortened scales with the scales of the original instrument amounted to about 0.90. The effects of the cross-cultural differences are the most obvious in the ImpSS, but they have been noted in the other dimensions as well. Besides the cross-cultural stability, research demonstrated a high criterion validity of dimensions of the ZKPQ. Namely, some studies revealed meaningful relationships between ZKPQ scales and risky and aggressive behavior (Joireman \& Kuhlman, 2004), as well as psychiatric symptoms (Wang et al., 2002). The studies of the relations between alternative FFM and other models showed expected convergence between dimensions of models from the same or different paradigms (Schmitz, 2004; Zuckerman et al., 1993; Aluja et al., 2002; Aluja et al., 2004).

The second criterion refers to the request that the basic personality traits must demonstrate at least moderate heritability (Zuckerman, 1991). For many years, behavioral genetic studies have mostly examined the heritability of Sensation Seeking. Heritability estimates of the SSS subscales have varied considerably. The lowest heritability has been shown for Boredom Susceptibility (48\% for men, $54 \%$ for women), while Experience Seeking has shown the highest heritability (62\% for men, 63\% for women) (Zuckerman, 
1994). In recent years, a twin study using the ZKPQ has been conducted. The results of the twin study on a German sample (Angleitner et al., 2004) suggested that the contribution of genetic factors in the explanation of the variance for the dimensions of the alternative FFM is about $50 \%$.

The third criterion has been based on the previous studies conducted on animals, which pointed to a possibility that the basic personality dimensions can be identified in some animal species (Zuckerman, 1991). This criterion is in line with the results of the numerous research on animal personality (Gosling, 2001; Gosling et al., 2003), which suggest that important individual differences in basic personality traits, such as dimensions of FFM or alternative FFM, can be measured in animal species.

The fourth criterion is that the important biological markers should be associated with basic personality dimensions. Each dimension of the alternative FFM has specific manifestations on a behavioral level, a base in the conditioning processes, as well as the physiological, biochemical, neurological, and genetic base. The interaction of the genes and the environment influences the structure and functioning of the brain system that underlies the trait. Individual differences in the system functioning influence the differences on the behavioral level (Matthews, 2004). However, although third and fourth criteria are very important parts of the alternative FFM, this research is not focused on their validation.

The examinations of the relations between PEN and alternative FFM, both derived from psychobiological approach, were mostly based on the hypothesis that it is possible to extract three latent higher order dimensions from those two models (Schmitz, 2004). Those dimensions are analogous to Eysenck's N, $\mathrm{E}$ and P. Latent dimension Neuroticism should include N from EPQ-R, NAnx and Agg-Host from ZKPQ. Extraversion should include E from EPQ-R, and Sy, Act and ImpSS and Agg-Host from ZKPQ. Psychoticism factor should include EPQ P, and ZKPQ Agg-Host and ImpSS. In general, results confirmed those expectations (Schmitz, 2004; Zuckerman et al., 1993; Aluja et al., 2002; Aluja et al., 2004).

The main aim of these studies is to examine the dimensions of the ZKPQ-50$\mathrm{CC}$ in light of some basic assumptions of the alternative FFM (Zuckerman, 1991). It concerns the stability of the model in different cultures, across samples of different genders and ages. As the part of process of construct validation of ZKPQ-50-CC we examined the convergence ZKPQ-50-CC dimensions with the PEN dimensions. 


\section{Study 1}

The basic aim of this research is the validation of the ZKPQ-50-CC (Aluja et al., 2006) in the Serbian culture. Previous studies showed the cross-cultural stability of the alternative FFM dimensions. Therefore, we expected that research in the Serbian culture will support the original factor structure of the questionnaire, and that the scales of the ZKPQ-50-CC will have good psychometric properties.

\section{Sample}

The research was conducted on the sample of 1155 participants - 511 men and 644 women. The subjects were 18 to 76 years old, of average age 32.11. The average age of the male participants is 33 , and the average age of the female participants is 31.4. The sample was selected using a non-probabilistic method from a population of undergraduate students of Universiy of Novi Sad, and a larger population of employed and retired persons who were examined within the project "Psychological Characteristics of the Society in Transition".

\section{Instrument}

Zuckerman-Kuhlman Personality Questionnaire-50-CC (ZKPQ-50-CC; Aluja et al., 2006) contains 50 true/false statements. The dimensions of this questionnaire are: Activity (ACT), Aggressiveness - Hostility (Agg-Host), Impulsive Sensation Seeking (ImpSS), Neuroticism - Anxiety (N-Anx) and Sociability (Sy). Each scale consists of 10 items. The translation of the Serbian version ZKPQ-50-CC involved a back translation procedure that showed consistency with the original English version.

\section{Results}

Results in general show acceptable psychometric properties (Table 1). The Alpha coefficients range from 0.65 for the Agg-Host to 0.78 for the N-Anx, while MSA ranges from 0.79 for the Agg-Host to 0.86 for the N-Anx. All dimensions, except the N-Anx, correlate significantly with age. T-tests with sex as grouping variable show significant differences only on Act and N-Anx. Men scored higher than women on Act, and women scored significantly higher on N-Anx. 
Table 1. Descriptive statistics (mean and standard deviation), measure of sampling adequacy, alpha and correlation with age of the ZKPQ-50-CC scales

\begin{tabular}{llllll}
\hline & M & SD & MSA & Alpha & $\begin{array}{l}\text { correlations } \\
\text { with age }\end{array}$ \\
\hline Act & 5.20 & 2.69 & 0.82 & 0.76 & $0.16^{*}$ \\
Agg/Host & 4.50 & 2.34 & 0.79 & 0.65 & $-0.12^{*}$ \\
ImpSS & 5.31 & 2.52 & 0.84 & 0.71 & $-0.32^{*}$ \\
N/Anx & 4.08 & 2.75 & 0.86 & 0.78 & 0.02 \\
Sy & 5.67 & 2.47 & 0.80 & 0.70 & $-0.34^{*}$ \\
\hline
\end{tabular}

$* \mathrm{p}<0.001$

Table 2. T-tests (grouping variable - sex; dependents - scores on the ZKPQ-50-CC scales)

\begin{tabular}{llllll}
\hline $\begin{array}{l}\text { Dependent } \\
\text { variables }\end{array}$ & $\begin{array}{l}\text { Male } \\
\text { mean } \\
(\mathrm{N}=511)\end{array}$ & $\begin{array}{l}\text { Female } \\
\text { mean } \\
(\mathrm{N}=644)\end{array}$ & $\mathrm{df}$ & $\mathrm{t}$-value & $\mathrm{p}$ \\
\hline Act & 5.49 & 4.97 & 1153 & 3.253 & 0.001 \\
Agg/Host & 4.56 & 4.45 & 1153 & 0.751 & 0.453 \\
Sy & 5.75 & 5.60 & 1153 & 1.006 & 0.314 \\
ImpSS & 5.41 & 5.22 & 1153 & 1.323 & 0.186 \\
$\mathrm{~N} /$ Anx & 3.08 & 4.89 & 1153 & -11.760 & 0.000 \\
\hline
\end{tabular}

A CFA was performed using SEPATH module of Statistica 7.1 (StatSoft, Inc., 2006). The estimation method was Maximum Likelihood. Each item was linked to a single factor. We tested three potentially viable models presented in Table 3. In the first model five latent variables were uncorrelated and in the second model (Figure 1) they were correlated. However, since items 6, 11, 19, and 43 loaded below .30 in the second model, we tested the third model, which excluded those items. The third model has the best indicators of fit.

Table 3. Goodness of fit indices of three models for the ZKPQ-50-CC scales

\begin{tabular}{lllllll}
\hline Model & $\mathrm{X}^{2}$ & $\mathrm{df}$ & $\mathrm{GFI}$ & $\mathrm{CFI}$ & $\mathrm{RMSEA}$ & $\mathrm{RMSSR}$ \\
\hline $\begin{array}{l}\text { Uncorrelated factors } \\
- \text { all items }\end{array}$ & 4209.93 & 1175 & 0.86 & 0.70 & 0.05 & 0.08 \\
$\begin{array}{l}\text { Correlated factors - } \\
\text { all items }\end{array}$ & 3801.66 & 1165 & 0.87 & 0.74 & 0.05 & 0.06 \\
$\begin{array}{l}\text { Correlated factors - } \\
\text { four items excluded* }\end{array}$ & 2966.02 & 979 & 0.89 & 0.78 & 0.04 & 0.05 \\
\hline
\end{tabular}


$\mathrm{GFI}=$ the goodness-of-fit index; adequate value above .90 ; liberal value above .85 $\mathrm{CFI}=$ comparative fit index; adequate value above .90

RMSEA = root mean squared error of approximation; adequate value below .10. RMSSR = RMS standardized residual; adequate value below .05 .

${ }^{*}$ Excluded items 6, 11, 19 and 43

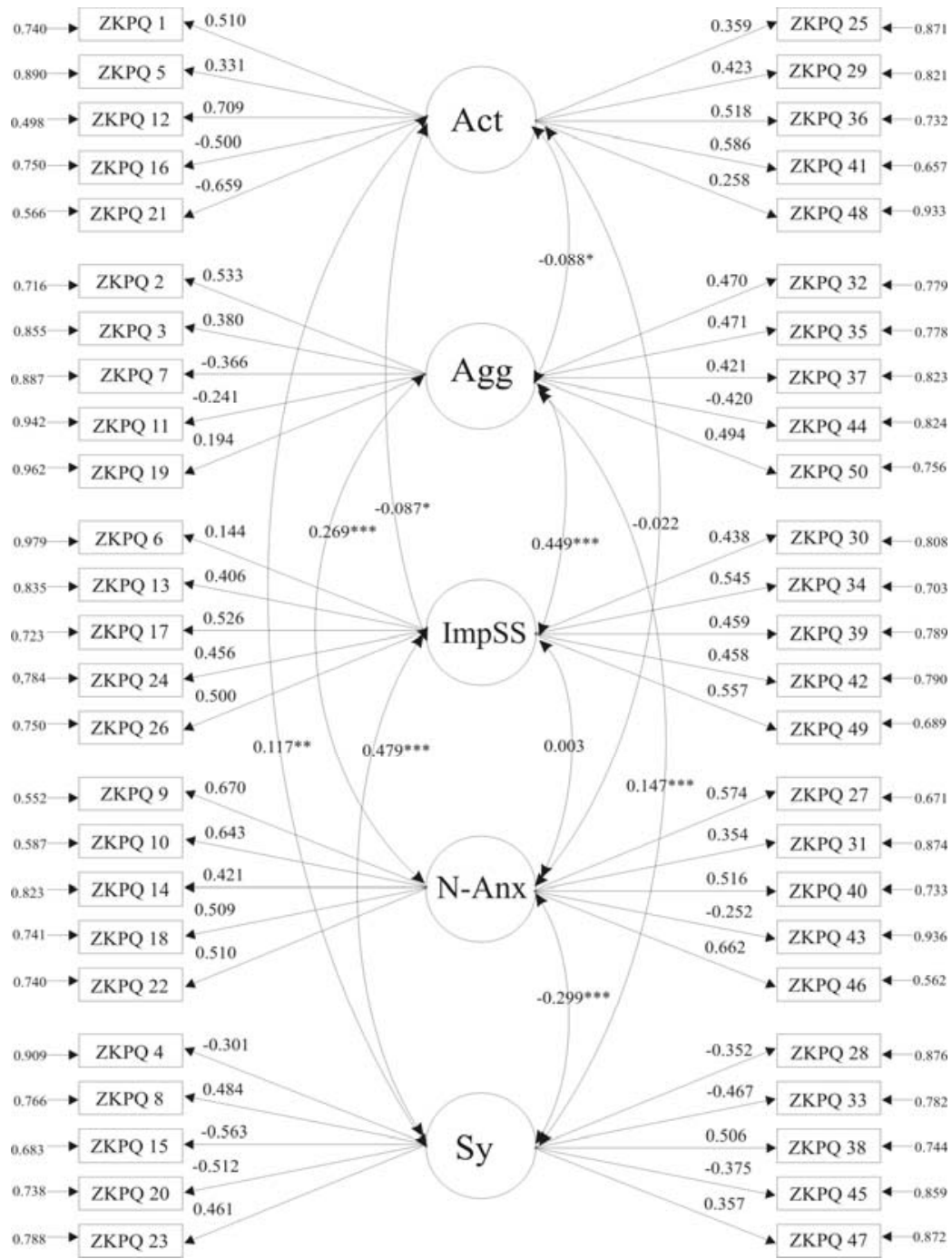

Fig. 1. Factor loadings resulting from the CFA on five factors model (the ZKPQ-50-CC scales are correlated)

${ }^{*} \mathrm{p}<.05 ;{ }^{* *} \mathrm{p}<.01 ;{ }^{* * *} \mathrm{p}<.001$; all factor loadings are significant at $\mathrm{p}<.001$ 
The findings regarding the correlations between the latent dimensions are mostly in accordance with the findings of the reference study. The correlations between Sy and other dimensions are positive in this study, contrary to the findings of the reference study.

\section{Discussion}

The basic aim of the research is the evaluation of the ZKPQ-50-CC (Aluja et al., 2006) on a Serbian sample. All scales have acceptable psychometric properties. The Serbian sample scored higher on Act and lower on Agg-Host compared to the American, German, Spanish and Swiss samples (Aluja et al., 2006). Certain differences in the age structure of the sample in our study and samples in previous studies (Aluja et al., 2006), could have some impact on differences in average scores. In previous studies with the ZKPQ and ZKPQ50-CC (Wu et al., 2000; Aluja et al., 2006), the results mostly showed low correlations between age and the dimensions of this model. In our research, the traits Sy and ImpSS correlate with age higher than usual. Again, the height of correlations could be influenced by the age structure of the sample. Act shows the unusual direction of association with age in comparison to the reference studies. The relationship between aging and the increase in Act scores could be explained by the effect of the tempo and the way of living imposed by the demands of adulthood, rather than by the temperamental dispositions of this dimension. The former explanation is supported by the fact that Act in the ZKPQ-50-CC contains, to a large extent, indicators of the Work Activity from the ZKPQ. This result suggests that the relations between age and the personality traits should be examined on the samples with the wider age range. On the other hand, research on the samples with the wider age range could face the question whether some changes are consequences of aging process or cohort effect.

The CFA almost entirely replicated the factor structure of the ZKPQ-50- CC (Aluja et al., 2006), except for four items. Item 6 originally belongs to Imp/SS, but in this factor solution loaded low on that factor. It is possible that such a result is an outcome of the ambiguous content of this item, which implies lack of control of the aggressive impulses in the Serbian culture, while in the American culture it implies the impulsive reaction, which is not necessarily related to aggressiveness. Similarly to the previous item, it is possible that the content of the item 43, which loaded poorly on N-Anx, was not properly understood by the participants. Clearly, the efforts to translate the items as closely to the original as possible resulted in the inclusion of some terms (a.k.a. "trivial"), 
which might have specific meanings in Serbian language. In light of these findings it seems necessary to revise the translation of these items.

Low loadings of the items 11 i 19 on Agg-Host could as well be due to cultural differences. The other possible explanation is that these items are not good measures of aggresiveness. Their content refers to different forms of behavior which could be related not only to aggressiveness, but also to some other basic personality traits. Perhaps the fact that this scale had worse psychometric properties than other scales of ZKPQ-50-CC, not only in our study, but also in the referent study (Aluja et al., 2006), reflects the problems with the item content of this scale.

\section{Study 2}

In the second study we tried to contribute to the process of construct validation of ZKPQ-50-CC (Aluja et al., 2006), by examination of its relations to EPQ-R (Eysenck et al., 1985). Both instruments are derived from psychobiological approach, with similar epistemological basis, but their authors conceptualized the basic personality dimensions in different levels of generality. So, theoretical and methodological implications of this part of research can be important for the construct validation process of both questionnaires.

\section{Sample}

The research included 200 subjects of both sexes - 90 men and 110 women. The subjects were from 19 to 63 yeas old. The average age was 37.11. This sample is one part of the larger sample from the first study. The sample is non-probabilistic and includes the participants who were administered both ZKPQ-50-CC and EPQ-R within the project "Psychological Characteristics of the Society in Transition".

\section{Instruments}

Zuckerman-Kuhlman Personality Questionnaire-50-CC (ZKPQ-50-CC; Aluja et al., 2006) and Eysenck Personality Questionnaire - Revised (EPQ-R, Eysenck et al., 1985) were applied. EPQ-R was translated to Serbian by Pero Šipka (Šipka, 1985). The questionnaire contains 103 items, with three scales measuring the dimensions of Eysenck's model - Neuroticism (23 items), Extraversion (21 items) and Psychoticism (38 items), along with the Lie scale which was not 
used in the analysis. The Alpha coefficients of reliability for EPQ-R scales are: $\alpha=0.85$ for Neuroticism, $\alpha=0.83$ for Extraversion, and $\alpha=0.66$ for Psychoticism.

\section{Results}

The relations between ZKPQ-50-CC and EPQ-R dimensions were evaluated using structural equation modeling. The models were tested using SEPATH, the SEM module in Statistica 7.1. The estimation method was Maximum Likelihood. Model fit was assessed using model chi-square, goodness of fit index (GFI), comparative fit index (CFI), the Root Mean Square Error of Approximation (RMSEA) and RMS standardized residual.

The first two models were based on the assumptions of the PEN model. Model 1 included $\mathrm{N}$ and $\mathrm{N}$-Anx on the first latent dimension, while Act, Sy and E were on the second latent dimension, and P, Agg-Host and ImpSS were attributed to the third latent dimension. The model assumed that the latent dimensions were uncorrelated. Model 2, with somewhat better fit, was the same as the first one, except that latent dimensions were correlated. Since the residual correlations between Agg-Host and N ( $\left.\mathrm{r}_{\text {res }}=0.185\right)$ and between AggHost and N-Anx ( $\left.r_{\text {res }}=0.143\right)$ were not negligible, Agg-Host was attributed to the first latent dimension. The loading of Act on the second latent dimension was not statistically significant $(\lambda=0.07 ; \mathrm{p}>0.1)$, but due to the residual correlation between Act and N-Anx $\left(\mathrm{r}_{\mathrm{res}}=-0.169\right)$, Act remained in the model and was included in the first latent dimension. Model 3 yielded slightly better fit, but the loading of Act on the first latent dimension was still insignificant $(\lambda=$ 0.092; $p>0.1$, which led to its exclusion from the model. Since some of the fit indices in the third model were still not fully acceptable, loadings on AggHost and ImpSS on the second latent dimension were included. Model 4 (Figure 2) had the best fit indices. It is important to mention that the attempts to include Act in the model resulted in extremely low and statistically insignificant loadings of this scale, no matter what dimension or dimensions it was attributed to. 
Dušanka Mitrović, Petar Čolović amd Snežana Smederevac

Table 4. Goodness of fit indices of four models

\begin{tabular}{llllllll}
\hline & $\mathrm{X}^{2}$ & $\mathrm{df}$ & $\mathrm{p}$ & $\mathrm{GFI}$ & $\mathrm{CFI}$ & $\mathrm{RMSEA}$ & $\mathrm{RMSSR}$ \\
\hline Model 1 & 106.940 & 21.000 & 0.000 & 0.901 & 0.778 & 0.126 & 0.136 \\
Model 2 & 48.943 & 17.000 & 0.000 & 0.944 & 0.917 & 0.094 & 0.071 \\
Model 3 & 48.068 & 17.000 & 0.000 & 0.944 & 0.92 & 0.094 & 0.069 \\
Model 4 & 11.652 & 8.000 & 0.167 & 0.984 & 0.99 & 0.048 & 0.034 \\
\hline
\end{tabular}

$\mathrm{GFI}=$ the goodness-of-fit index; adequate value above .90 ; liberal value above .85

$\mathrm{CFI}=$ comparative fit index; adequate value above .90

RMSEA = root mean squared error of approximation; adequate value below .10.

RMSSR = RMS standardized residual; adequate value below .05 .

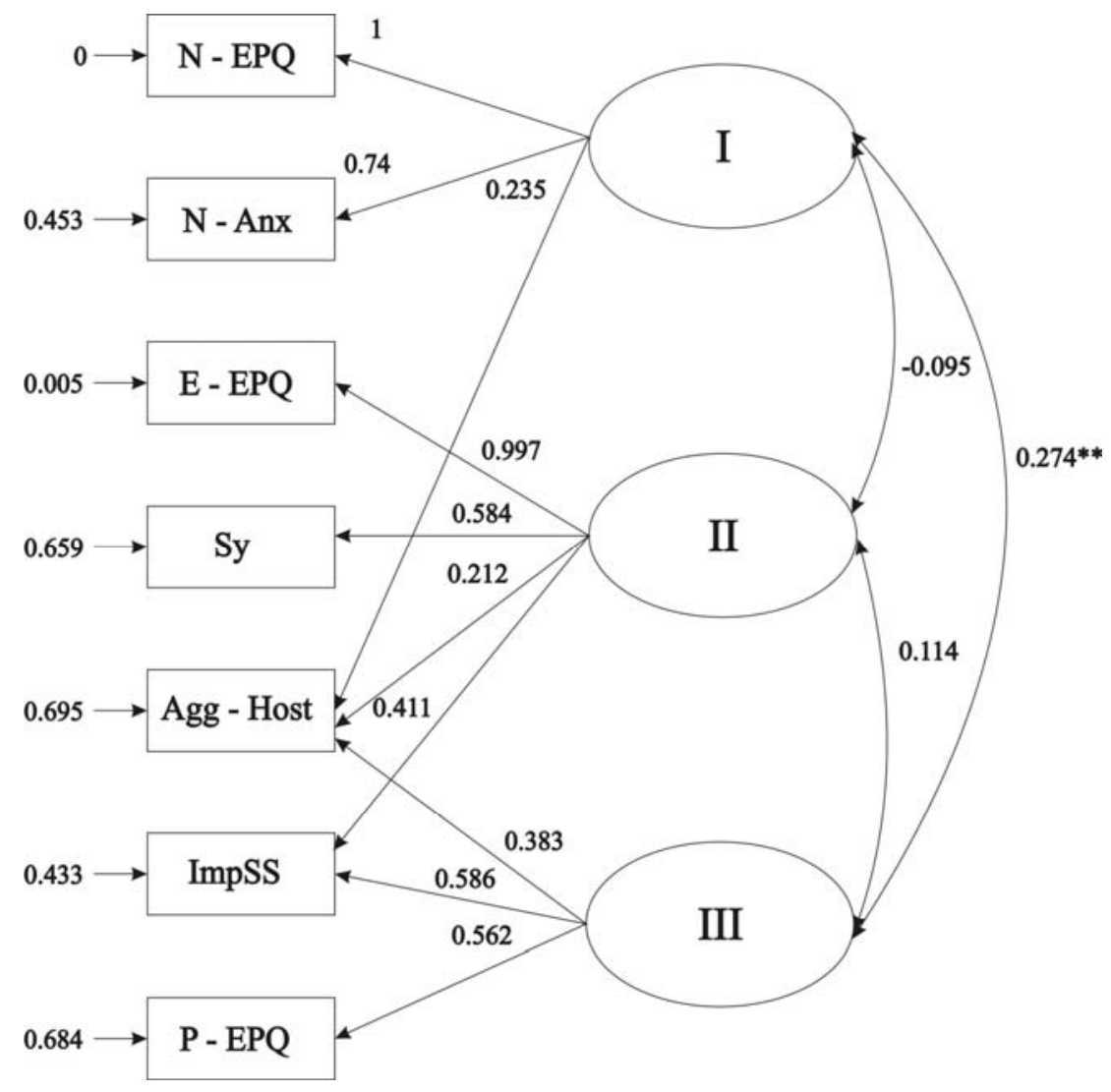

Fig. 2. Parameter estimates for model four

** $p<.01$; all factor loadings significant at $p<.001$ 


\section{Discussion}

To date, very little research has considered the construct validity of the ZKPQ-50-CC. The current study aimed to examine relations between ZKPQ50-CC and EPQ-R scales. In general, our results were congruent with results of previous studies with ZKPQ (Schmitz, 2004; Zuckerman et al., 1993; Aluja et al., 2002; Aluja et al., 2004). However, few unexpected loadings of some scales appeared.

The structure of the first latent dimension corresponds to the general neuroticism, with $\mathrm{N}$ from EPQ-R loading the highest. N-Anx from ZKPQ-50-CC loads high as well. $\mathrm{N}$ from PEN accounts for negative affectivity, including guilt, anxiety, loneliness, while N-Anx is predominantly defined by the indicators of non-specific tension increase. Agg-Host also significantly loads on this latent dimension. The results of previous studies regarding the position of aggressiveness were contradictory. In Zuckerman's (Zuckerman et al.., 1993) research with similar conceptual and methodological premises, the Agg-Host had a significant contribution in all three dimensions. Accordingly, the saturation with $\mathrm{P}$ was 0.63 , and with $\mathrm{E}$ it was 0.27. Schmitz's results (Schmitz, 2004 ) showed that the aggression is related only to $P(0.13)$ and $N(-0.38)$. These results suggest that some aspects of aggressiveness can be included in broader construct of negative emotionality, which corresponds to neuroticism.

The second latent variable corresponds to the extraversion. Extraversion includes E from EPQ-R and Sy, ImpSS and Agg-Host from ZKPQ-50-CC. Act does not significantly participate in the structure of this (nor the other) latent variables. According to the theoretical assumptions, Act is a part of the Extraversion domain along with Sy. However, the content of Act in ZKPQ-50$\mathrm{CC}$ includes predominantly indicators of Work Activity from ZKPQ, and only few indicators of temperamental dispositions. In other words, it is possible that $\mathrm{E}$ and Act do not have much in common.

As expected, P and Agg-Host load high on the third dimension, which corresponds to psychoticism. ImpSS loaded on the second and the third latent dimension. Obviously some aspects of ImpSS are connected with E, and some with P. It is possible that stimulation seeking can be as important feature of the extraversion as the need for affiliation. But, some aspects of ImpSS can be connected with the lack of control and tendency of risky behavior, which are the most important caracteristics of $\mathrm{P}$.

Correlations between three latent dimensions are the most similar to Eysenck's assumptions (Eysenck, 1990), which are not always replicated in 
studies. Although significant correlations between $\mathrm{E}$ and $\mathrm{N}$ were shown in some studies (Schmitz, 2004), results are usually inconsistent. Correlation of 0,27 between $\mathrm{N}$ and $\mathrm{P}$ emphasizes similarities of two aspects of negative emotionality. Namely, it is possible that negative emotionality accounts for some aspects of aggressiveness. This result is congruent with Zuckerman's (Zuckerman, 1991) assumptions on biological basis and descriptive aspects of Neuroticism.

\section{General discussion}

The basic aim of the studies presented in this paper was the evaluation of the alternative FFM in light of some fundamental initial assumptions of the author (Zuckerman, 1991). The first Zuckerman's criterion for the conceptualization of personality traits as basic refers to the possibility of their reliable identification by applying different methods, on the samples of different sex, age and cultures. In that context, the results of this research support the conceptualization of the alternative FFM dimensions as basic. Namely, those dimensions were appropriately replicated in the Serbian culture, on the sample of participants of different sex and age. In light of satisfactory structural and psychometric features of the ZKPQ-50-CC on the Serbian sample, the results may be considered as a contribution to evidence accumulation for the applicability of the alternative FFM in different cultures. The comparison of ZKPQ-50-CC scales to the measures of personality dimensions of Eysenck's model mostly showed expected relations, with the exception of Act scale.

\section{References}

Aluja, A., Garcia, O., \& Garcia, L.F. (2002). A comparative study of Zuckerman 's three structural models for personality through the NEO-PI-R, ZKPQ-III-R, EPQ-RS, and Goldberg's 50-bipolar adjectives. Personality and Individual Differences, 33, 713 - 725.

Aluja, A., Garcia, O., \& Garcia, L.F. (2004). Replicability of the three, four and five Zuckerman's personality super-factors: exploratory and confirmatory factor analysis of the EPQ-RS, ZKPQ and NEO-PI-R. Personality and Individual Differences, 36, 1093-1108.

Aluja, A., Rossier, J., Garcia, L.F., Angleitner, A., Kuhlman, M., \& Zuckerman, M. (2006). A cross-cultural shortened form of the ZKPQ (ZKPQ-50-cc) adapted to English, French, German, and Spanish languages. Personality and Individual Differences, 41, 619 - 628. 
Eysenck, H. J. (1990). Biological dimensions of personality. In L. A. Pervin (Ed.), Handbook of personality: Theory and research (pp. 244-276). New York: Guilford.

Eysenck, H. J., Eysenck, S. B. G. \& Barrett, P. (1985). A revised version of the psychoticism scale. Personality and Individual Differences, 6, 21-29.

Gosling, S. D. (2001). From Mice to Man: What Can We Learn About Personality From Animal Research? Psychological Bulletin, 127, 45-86.

Gosling, S. D., Kwan, V. S., \& John, O. P. (2003). A Dog's Got Personality: A Cross-Species Comparative Approach to Personality Judgments in Dogs and Humans. Journal of Personality and Social Psychology, 85, 1161-1169.

Joireman, J. \& Kuhlman, D. M. (2004). The Zuckerman-Kuhlman Personality Questionnaire: Origin, Development and Validity of Measure to Assess an Alternative Five-Factor Model of Personality. In R.M. Stelmack (Ed). On ThePsychobiology of Personality: Essays in Honor of Marvin Zuckerman (pp.49 - 65). Amsterdam: Elsevier.

Matthews, G. (2004). Neuroticism from the Top Down: Psychophysiology and Negative Emotionality. In R.M. Stelmack (Ed). On ThePsychobiology of Personality: Essays in Honor of Marvin Zuckerman (pp.249 - 266). Amsterdam: Elsevier.

Schmitz, P. G. (2004). On the Alternative Five-Factor Model: Structure and Correlates. In R.M. Stelmack (Ed). On ThePsychobiology of Personality: Essays in Honor of Marvin Zuckerman (pp.65 - 87). Amsterdam: Elsevier.

Šipka, P. (1985). Prevod i adaptacija Eysenckovog testa ličnosti EPQ-R. Beograd: Vojnomedicinska akademija.

Wang, W., Cao, M., Zhu, S., Gu, J., Liu, J., \& Wang, Y. (2002). ZuckermanKuhlman's Personality Questionnaire in patients with major depression. Social behavior and personality, 30, 757-764.

Wu, Y. -X., Wang, W., Du, W.Y., Li, J., Jiang, X.-F., \& Wang, Y.-H. (2000). Development of a Chinese version of the Zuckerman-Kuhlman personality questionnaire: reliabilities and gender/age effect. Social Behaviour and Personality, 28, 241 - 250.

Zuckerman, M. (1991). Psychobiology of Personality. Cambridge: Cambridge University press.

Zuckerman, M. (1994). Behavioral Expressions and Biosocial Bases of Sensation Seeking. Cambridge, Cambridge University Press. 
Zuckerman, M. (2002). Zuckerman-Kuhlman Personality Questionnaire (ZKPQ): An alternative five factorial model. In B. DeRaad, and M. Perusini (Eds.), Big Five Assessment (pp. 377 - 396). Seattle: Hogrefe and Huber Publishers.

Zuckerman, M., Kuhlman, D. M., Joireman, J., Teta, P., \& Kraft, M. (1993). A comparison of three structural models for personality: the big three, the big five, and the alternative five. Journal of Personality and Social Psychology, 65, 757 - 768.

Primljeno: 10. 11. 2009; prihvaćeno za štampu: 4.12. 2009. 